\title{
Analysis of ECM Phenomena with Equivalent Circuit for Electrolysis
}

\author{
Wataru Natsu*, Takashi Kunimi* \\ (Received Sept. 20, 2009)
}

* Tokyo University of Agriculture and Technology, 2-24-16 Nakacho, Koganei, Tokyo 184-8588, Japan

\begin{abstract}
We investigated the interaction between the tool-electrode and workpiece in electrochemical machining (ECM) by establishing an equivalent circuit model for electrolysis. A Zener diode was introduced for the first time to account for the fact that Faradaic current only starts to flow after the potential across the double layer at the solid and liquid interface exceeds the threshold of the electrolytic reaction. After determining the circuit parameters by comparing experimental and analytical results, we examined the ECM characteristics, the Faradaic current as well as the influence of the pulse-off time on the machining accuracy and removal rate. It was found that our model incorporating the Zener diode accurately describes the ECM process. From the simulation, the pulse-off time was found to have a significant impact on machining accuracy.
\end{abstract}

Key words: ECM, Equivalent circuit, Faradaic current, Machining accuracy, Removal rate

\section{INTRODUCTION}

Electrochemical machining (ECM) is a method used to shape metallic workpieces by electrochemically removing or adding material. Since it is an electrolytic process, there are no heat affected zones, residual stresses, cracks or burrs on the machined surface. This contrasts with thermal and mechanical processing methods. On the other hand, since processing current flows even if the gap length between the tool electrode and the workpiece is large, ECM has not been considered suitable for microfabrication.

High-accuracy ECM was realized using an ultrashort pulse power supply. Rolf Schuster et al. ${ }^{1)}$ reported that local etching of copper and silicon becomes possible by applying ultrashort voltage pulses, since the electrochemical reactions are then confined to regions in proximity to the electrodes. Considerable research on micro-ECM using ultrashort pulses, and other techniques, has been reported 23344). Nevertheless, one drawback to micro-ECM is the low removal rate.

Establishing a suitable equivalent circuit is helpful for understanding the ECM phenomena, especially the influence of ECM conditions, since the performance of an electrochemical cell can usually be represented by an equivalent circuit. For example, a simple equivalent circuit with a resistor and a serially connected capacitor between two electrodes has been used to explain local etching with ultrashort pulses ${ }^{1)}$. Although this model can qualitatively explain the principle of ECM with ultrashort pulses, it is insufficient for representing machining characteristics, since there is no Faradaic impedance in the circuit. One the other hand, although several equivalent circuits with the Faradaic impedance in parallel with a double layer capacitor ${ }^{5(6)}$ have been proposed, these models show that the electrochemical reaction occurs as soon as the pulsed power is applied, which does not correspond to the actual phenomena.

In order to solve the above problems and determine the effects of ECM conditions on machining accuracy and removal rate, a Zener diode is introduced for the first time in the equivalent circuit. This approach allows the phenomena to be modeled such that the processing current only starts to flow after the potential difference across the electrochemical double layer at the solid-liquid interface exceeds the threshold of the electrolytic reaction. Using this circuit, not only the potential difference across the electrochemical double layer but also the current flow through the Faradaic impedance can be theoretically analyzed. After determining the parameters of the equivalent circuit by comparing the experimental and analytical results, we examine the ECM characteristic, the Faradaic current, the influence of the pulse-off time on machining accuracy, as well as the removal rate.

\section{ESTABLISHMENT OF ECM EQUIVALENT CIRCUIT}

In ECM, the electrochemical reactions on the surface of the tool electrode and on the workpiece occur after the potential difference across the electrochemical double layer at the liquid-solid interface reaches the threshold. In this study, this 
phenomenon is modeled with a Zener diode whose breakdown voltage is equal to the threshold voltage. The proposed equivalent circuit for the ECM cell is shown in Figure 1. A serially connected Zener diode and Faraday resistance are in parallel with a double-layer capacitor. As a result, current flows through the Faraday resistance not immediately after the pulsed voltage is applied, but only once the voltage across the double-layer capacitor exceeds the breakdown voltage of the Zener diode. Part A in Figure 1 represents the interface between the workpiece surface and the electrolyte, part B represents the bulk electrolyte and part $\mathrm{C}$ represents the interface between the tool electrode and the electrolyte. Here, $C_{d l}$ is the double layer capacitance, $R_{f}$ is the Faraday resistance through which the processing current flows, $D_{z}$ is the Zener diode introduced in the our ECM model, and $R_{e}$ is the electrolyte resistance between the two electrodes.

Strictly speaking, the parameters in parts A and C are different since the reactions at these two interfaces differ. However, for simplicity we assume both parts to be the same.

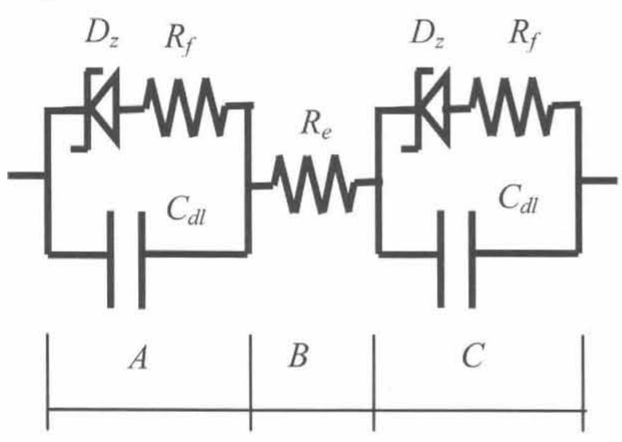

Fig. 1 Equivalent circuit for ECM cell

\section{IDENTIFICATION OF PARAMETERS}

\subsection{Outline of experimental method}

The experimental device shown in Figure 2 was produced to identify the parameters for the equivalent circuit and examine the ECM process. Because the three electrode-workpiece pairs with different gap lengths are connected in parallel, the relation between gap length and the current waveform can be determined. We measure the current through the resistance $R_{m}(0.1 \Omega) . R_{l}(5 \Omega)$ is the resistance used to limit the maximum current and protect the power supply. Titanium and stainless cylindrical bars of 3 $\mathrm{mm}$ in diameter are used as the tool-electrode and workpiece, respectively. The side surface of each cylinder is insulated with silicon tubes so as to confine the current flow through the end facets. Finally, the electrolyte is a $5 \mathrm{wt} \% \mathrm{NaNO}_{3}$ solution.
During extrapolation of the equivalent circuit parameters, only one electrode-workpiece pair shown in Figure 2 is connected to the power supply. All three pairs are connected when studying machining accuracy and removal rate.

\subsection{Identification of circuit parameters}

Unknown parameters in the circuit, namely, $C_{d l}, R_{f}$, $D_{z}$ and $R_{e}$, are identified by comparing the calculated and experimentally measured currents flowing through $R_{m}$ under different pulse conditions. Here, only one electrode-workpiece pair is connected to the power supply. The circuit corresponding to the experimental setup is shown in Figure 3, where $C_{s}$ is the stray capacitance attributed to wiring. Experimental conditions are listed in Table 1.

Examples of the voltage waveform of the applied pulse and of the measured waveform of the total current flowing through the resistance $R_{m}$ are shown in Figure 4. By applying pulses with different voltages, the corresponding current waveforms were obtained. Figure 5 shows the current $i_{n}$ prior to an

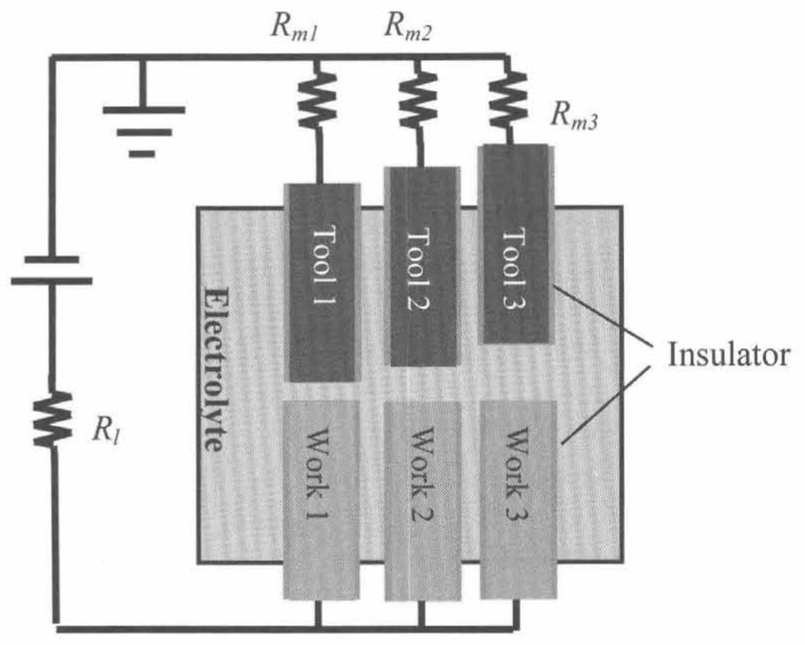

Fig.2 Schematic of experimental setup

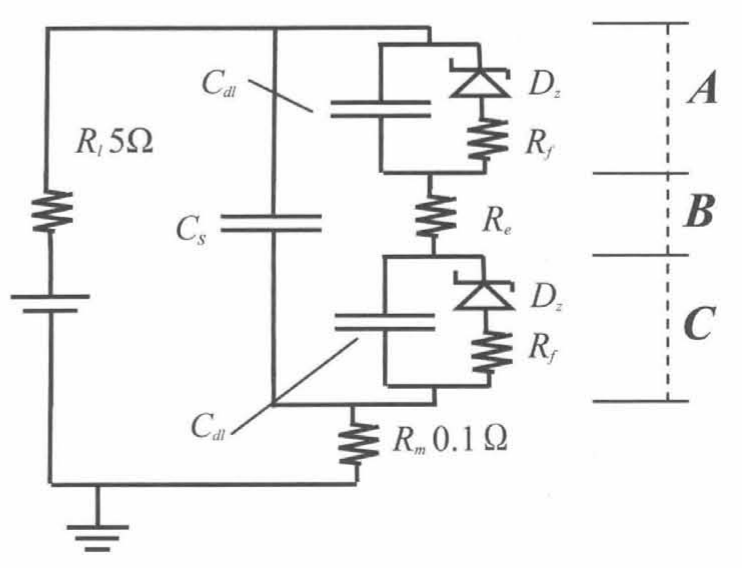

Fig.3 ECM circuit model 
Table 1 Experimental conditions

\begin{tabular}{|c|c|}
\hline Workpiece material & SUS304 \\
\hline Tool electrode material & $\mathrm{Ti}$ \\
\hline Pulse duty & $50 \%$ \\
\hline Pulse width & $5 \mu \mathrm{s}$ \\
\hline Gap length & $0.1 \mathrm{~mm}$ \\
\hline Electrolyte & $\mathrm{NaNO}_{3}$ \\
\hline Electrolyte density & $5 \%$ \\
\hline
\end{tabular}

input voltage pulse, and the current $i_{p}$ just before the pulse is cut off. The slope of the current $i_{n}$ changes for a voltage value $v_{\text {rea }}$ around $5 \mathrm{~V}$, although the slope of the current $i_{p}$ remains constant. One explanation is that when the applied voltage is smaller than $v_{\text {rea }}$, the potential difference across of the electrochemical double layer is always smaller than the threshold value for the electrolytic reaction. In such a case, there is no Faradaic current, and nearly all the electric charge accumulated in the double layer during pulse-on time is discharged in the opposite direction when the pulse is turned off. This can be seen in Figure 4, where the current $i_{n}$ is almost equal to $i_{p}$. When the applied voltage exceeds $v_{\text {rea }}$, the potential difference across the electrochemical double layer exceeds the threshold value, which causes the current to begin flowing through the Faraday resistance. In this case, part of the accumulated charge flows through the Faraday resistance, which causes $i_{n}$ to have a smaller absolute value than $i_{p}$.

Figure 6 shows the experimentally observed change in $i_{p}$ and $i_{n}$, while the gap length between the electrode and workpiece is varied from 0.1 to $1.0 \mathrm{~mm}$. Here, the power supply pulse voltage is fixed to $10 \mathrm{~V}$. Since it is reasonable to assume that the change in gap length only affects the overall bulk electrolyte resistance, we can infer the relationship between gap length and resistance.

To determine the equivalent circuit parameters, we used the dependence of $i_{p}$ and $i_{n}$ on both the applied voltage and the gap length. Moreover, time-dependent current curves were also taken into account. After extrapolating the circuit parameters, the simulated current waveforms were compared to the experimental ones. The parameters were then adjusted over successive iterations so as to obtain the closest possible agreement with the experimental waveform. Current waveforms for different voltages were calculated using "Switcher CAD 3" simulation software (Linear Technology Corporation). Table 2 lists the obtained equivalent circuit parameters, where $V_{b}$ is the breakdown voltage of $D_{z}$, and $g$ is the gap leght. The simulated and measured currents were in good agreement, as shown in Figure 7, with some differences during rise and fall times.

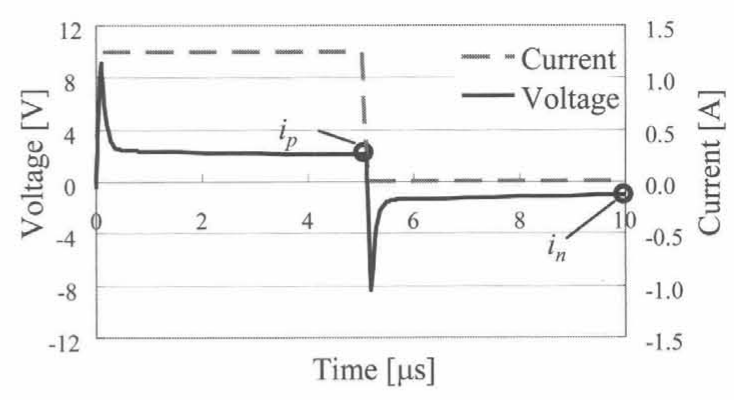

Fig.4 Applied voltage and measured current

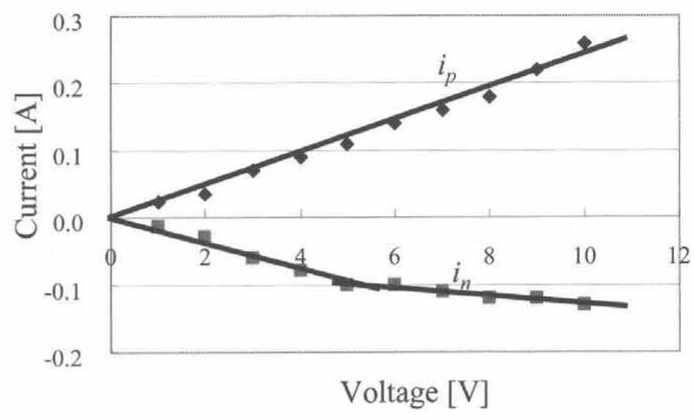

Fig.5 Relationship between power supply voltage and measured current

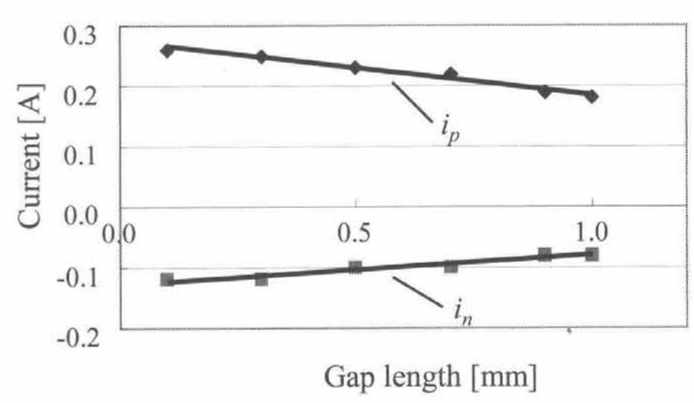

Fig.6 Relationship between gap length and measured current

Table 2 Identified parameters

\begin{tabular}{|c|c|}
\hline$C_{d l}$ & $2 \mu \mathrm{F}$ \\
\hline$R_{e}$ & $7.5 g+17.25 \Omega$ \\
\hline$R_{f}$ & $7 \Omega$ \\
\hline$V_{b}$ & $1.25 \mathrm{~V}$ \\
\hline$C_{s}$ & $0.02 \mu \mathrm{F}$ \\
\hline
\end{tabular}

$g$ : gap length

\section{ANALYSIS OF ECM CHARACTERISTICS WITH PROPOSED EQUIVALENT CIRCUIT}

On the basis of the equivalent circuit proposed in this research, we observe and discuss the time change 
in the potential difference across the double layer and in the Faradaic current, as well as the influence of pulse-off time on the machining accuracy and removal rate.

\subsection{Observation of double-layer potential and Faradaic current}

The change in the potential difference across the double layer and the Faradaic current is analyzed by using the equivalent circuit with the extrapolated parameters. The pulse on and off durations are both 5 $\mu$ s, with peak voltages of $3 \mathrm{~V}, 5 \mathrm{~V}$ and $10 \mathrm{~V}$. Currents flowing through the resistance $R_{m}$, currents through the double layer and the Faradaic current are calculated and shown in Figures 8, 9 and 10, respectively.

First, it is important to consider the results for an input voltage of $5 \mathrm{~V}$ in each figure. When this input voltage is applied, the capacitor becomes charged and the potential difference across it rises. The Faradaic current flow appears after the voltage across the double layer exceeds the breakdown voltage $V_{b}$ $(=1.25 \mathrm{~V})$ of the Zener diode. When the pulse is turned off, the capacitor starts to discharge and its voltage falls. Faradaic current does not flow when the potential difference across the double layer falls below the breakdown voltage $V_{b}$.

On the other hand, the Faradaic current is always present for an input voltage of $10 \mathrm{~V}$, since the potential across the double layer is always higher than $V_{b}$. Conversely, for an input voltage of $3 \mathrm{~V}$, since the potential across the double layer never exceeds $V_{b}$, no Faradaic current is present in the circuit.

From these results, we can conclude that the Faradaic current, which is responsible for the electrolytic reaction, is governed by the potential across the double layer, which itself is driven by the charging and discharging process.

\subsection{Influence of pulse-off time on machining accuracy and removal rate}

Previous studies on the effects of pulse-on time on machining accuracy have employed a simple equivalent circuit model ${ }^{1)}$ that did not allow the influence of pulse-off time to be examined.

In this section, the effects of pulse-off time on machining accuracy and removal rate are examined by means of the newly established equivalent circuit. Machining accuracy is evaluated through the relationship between the Faradaic current and gap length. Specifically, the more the Faradaic current changes with gap length, the higher the machining accuracy becomes.

The amount of material removed from the workpiece (hereinafter, workpiece removal amount)

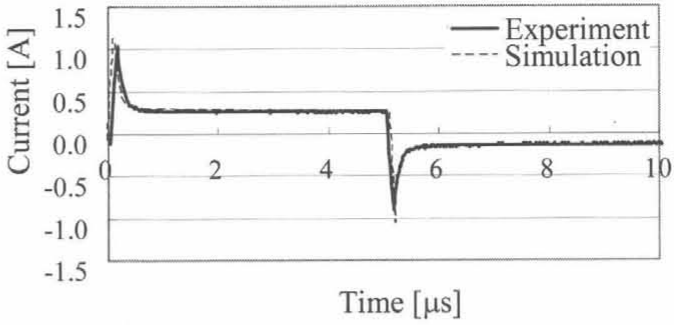

Fig.7 Waveform of experimental and simulated result

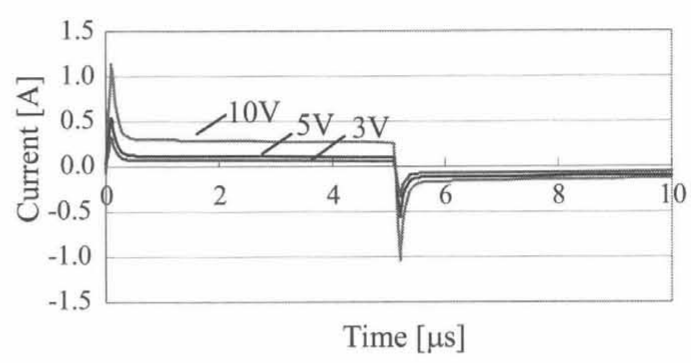

Fig.8 Calculated current flowing through $R_{m}$

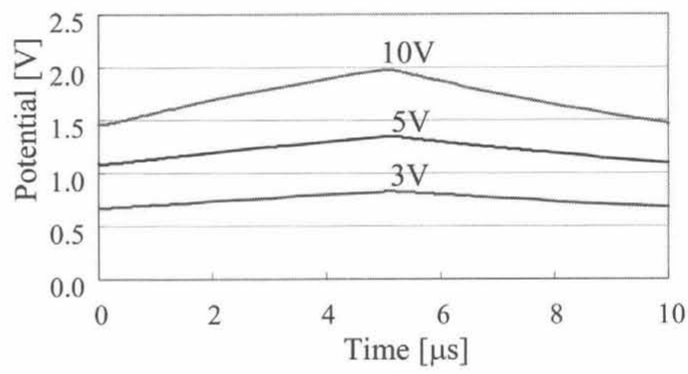

Fig.9 Waveform of potential cross double layer

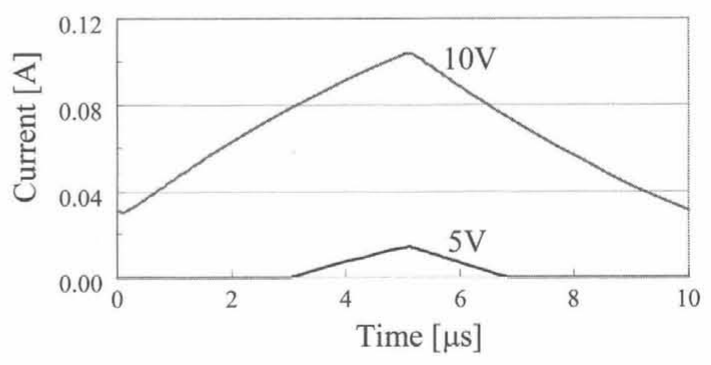

Fig.10 Waveform of Faradaic current

is proportional to the current flow through the Faradaic resistance, and can be expressed by Eq. (1). Here, $m$ is the workpiece removal amount, $\eta$ is the current efficiency, $A$ is the atomic weight, $Q$ is the charge, $I(t)$ is the Faradaic current, $t$ is the duration, $n$ is the valence and $F$ is the Faraday constant.

We consider three electrode-workpiece pairs, as shown in Figure 2, with respective gap lengths of 0.2 , 


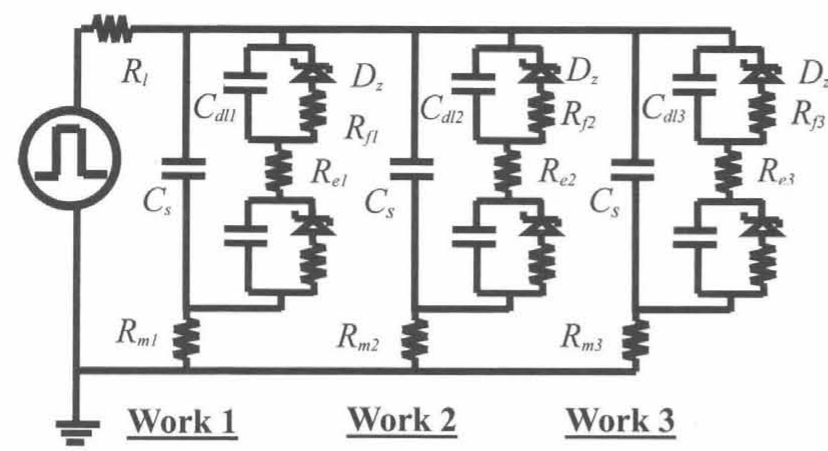

Fig. 11 Circuit model for different gap lengths

$$
\begin{aligned}
& m=\eta \frac{A Q}{n F}=\int \eta \frac{A I(t)}{n F} d t \\
& Q^{\prime}=\frac{Q_{1}-Q_{3}}{Q_{1}} \\
& W^{\prime}=\frac{W_{1}-W_{3}}{W_{1}}
\end{aligned}
$$

0.3 and $0.5 \mathrm{~mm}$. The corresponding equivalent circuit is shown in Figure 11. Here, the values of $R_{e l}, R_{e 2}$ and $R_{e 3}$ for the three gap lengths are, respectively, $19.5 \Omega$, $21 \Omega$ and $24 \Omega$, as per the results listed in Table 2 . Other circuit parameters are also taken from Table 2. We then calculate the charges $Q_{1}, Q_{2}$ and $Q_{3}$ that pass through the three respective Faraday resistances, $R_{l}$, $R_{2}$ and $R_{3}$. We calculate the charge ratio $Q^{\prime}$ using Eq. (2). The larger the charge ratio is, the greater the difference between $Q_{1}$ and $Q_{3}$, which corresponds to higher machining accuracy.

For different pulse-off times, charges $Q_{1}, Q_{2}$ and $Q_{3}$, as well as the charge ratio, are determined. Here, we consider $10 \mathrm{~V}$ pulses with a fixed $5 \mu$ s pulse-on time, while the pulse-off time is varied from $1 \mu$ s to $20 \mu \mathrm{s}$. For a pulse-off time of $17 \mu \mathrm{s}$, the simulated potentials across the double layer as well as the Faradaic currents for the three electrode-workpiece pairs are shown in Figure 12. Comparing Figure 12 with Figure 9, it can be seen that as the pulse-off time increases, the potential across the double layer decreases, and the period without Faradaic current increases. Greater gap lengths shorten the aforementioned period.

The calculated charges and charge ratios for different pulse-off times are shown in Figures 13 and 14. We use the charge to evaluate the removal rate, while the charge ratio is used to evaluate the machining accuracy. The results show that a long pulse-off time is required for high machining accuracy, although at the expense of the removal rate.
To verify our simulation results, machining experiments were carried out under conditions corresponding to the simulation parameters. The workpiece removal amount was measured after the machining process, and the ratio of the workpiece removal amount was obtained using Eq. (3), where $W_{1}$ and $W_{3}$ are the removal amounts of workpieces 1 and 3, respectively. The results are shown in Figures 15 and 16. It was found that a longer pulse-off time leads to higher machining accuracy and lower removal rate. Therefore, our experimental results are in good agreement with the simulation results.

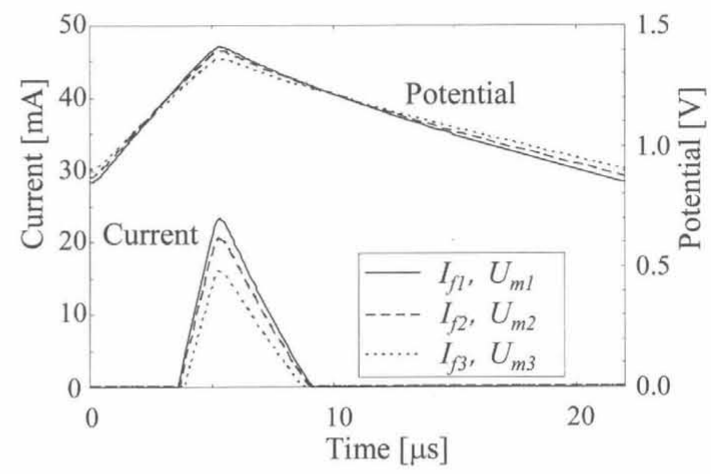

Fig. 12 Waveform of Faradaic current and potential across double layer

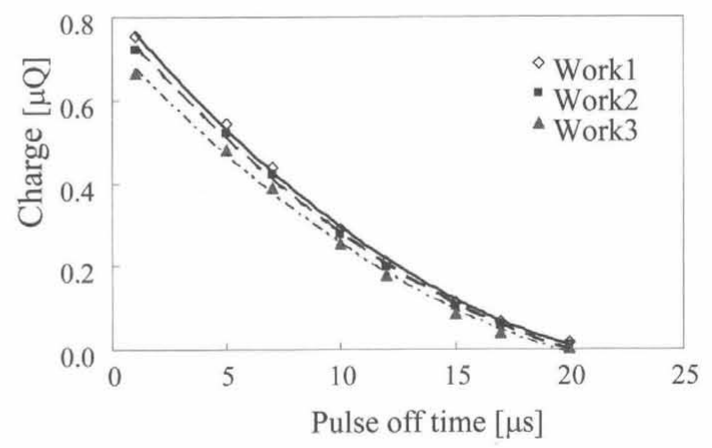

Fig.13 Relation between pulse-off time and charge

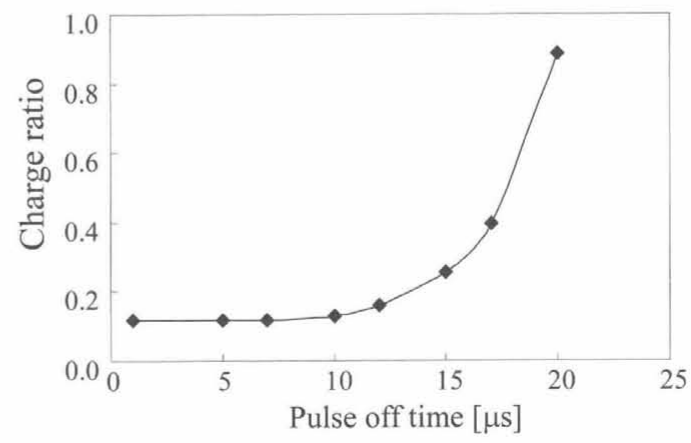

Fig.14 Relation between pulse-off time and charge ratio 


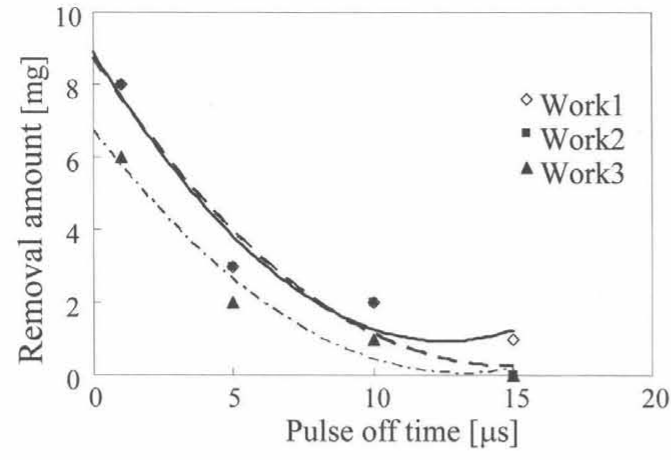

Fig.15 Relation between pulse-off time and workpiece removal amount

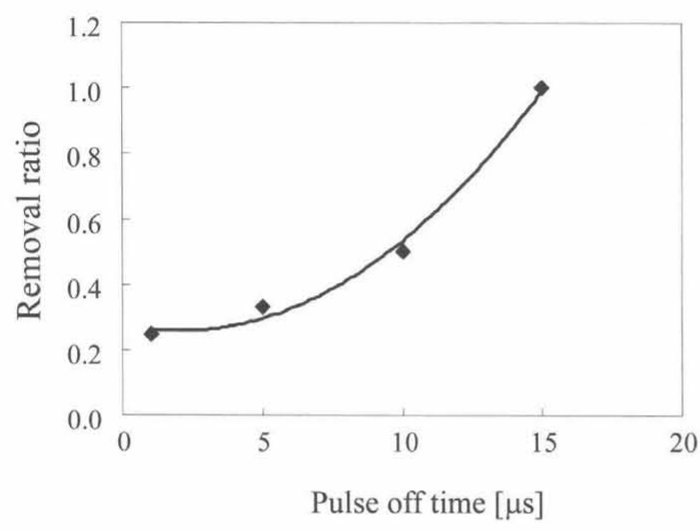

Fig.16 Relation between pulse-off time and removal ratio

\subsection{Discussions on use of equivalent circuit}

Based on the above analysis and experimental verification, we can conclude that the newly established ECM equivalent circuit is effective for investigating the machining process and analyzing the relationship between machining accuracy and removal rate. However, owing to the complexity of ECM and its dependence on factors such as the inter-electrode medium, temperature, product materials and bubbles, the machining process cannot be perfectly modeled with the equivalent circuit. Moreover, the current efficiency and the valence are needed to precisely obtain the removal amount. These limitations must be taken into consideration when using this equivalent circuit.

\section{CONCLUSIONS}

In this paper, we proposed an equivalent circuit for ECM, which can be used to analyze machining processes. The parameters in the equivalent circuit were extrapolated by comparing simulated and measured currents. The influence of pulse-off time on machining accuracy and rate were simulated and verified experimentally. We concluded that the pulse-off time plays an important role in machining accuracy.

\section{References}

1) Rolf Schuster, Viola Kirchner, Philippe Allongue, Gerhard Ertl: Electrochemical Micromachining, Science, 289(5476), (2000), pp.98-101.

2) B Bhattacharyya and J Muda: Experimental investigation into electrochemical micromachining (EMM) process, Journal of Materials Processing Technology 140(1-3), (2003), pp.287-291.

3) B.H. Kim, C.W. Na, Y.S. Lee, D.K. Choi, C.N. Chu: Micro Electrochemical Machining of 3D Micro Structure Using Dilute Sulfuric Acid, CIRP Annals - Manufacturing Technology, 54(1), (2005), pp.191-194.

4) K.P. Rajurkar, G. L., A. Malshe: Micro and nano machining by electro-physical and chemical processes, Annuals of the CIRP, 55(2), (2006), pp. 643-666.

5) A. J. Bard, L. R. Faulkner: Electrochemical methods (second edition), John Wiley \& Sons, INC., (2001), pp.376.

6) J. A. Kenney: Transient Charging Processes at Liquid-Solid and Vacuum-Solid Interfaces, The PhD Dissertation of University of Texas at Austin, (2006), pp.16. 